\title{
AUTHOR INDEX VOLUME 1 (2008)
}

Alias, N. A. 195

Ananta, S. 229

Arof, A. K. 195

Bae, I.-S. 77

Boo, J.-H. 77

Brunel, L. C. 7

Chang, C. W. 183

Chang, H. W. 183

Chang, W. C. 183

Chen, I. W. 183

Chen, J. L. 115

Chen, X. 253

Cho, S.-J. 77

Corkovic, S. 13

Dai, X. F. 115

Ding, J. 189

Drahus, M. D. 7

Eichel, R.-A. 7

Erdem, E. 7

Eres, G. 71

Feng, Y. P. 1

Geohegan, D. B. 71

Guo, J. 71

Hayashi, A. 31

Hodgson, P. D. 215

Hoffmann, M. J. 7

Hong, L. 59

Huang, S. T. 183

Ji, W. 1

Jiang, C. B. 115

Kang, M. S. 151

Khamman, O. 229

Kim, J. I. 209

Kim, J. M. 151

Kim, S. S. 151

Kim, Y. J. 83

Kim, Y. W. 145, 203

Kitamura, K. 177

Kong, S. S. 151

Kungl, H. 7
Lai, M. O. 253

Lee, J. H. 65

Lee, J. M. 145

Lee, Y. H. 145

Lee, Y. J. 209

Leisten, O. P. 25

Li, T. 225

Li, Y. 215

Li, Z. H. 139

Lim, S. H. 1

Lin, J. 1

Liu, B. 173

Liu, L. 1

Liu, S. Y. 59

Liu, X. X. 253

Liu, X. 177

Liu, Z. 55

Lu, L. 121, 253

Luo, H. Z. 115

Ma, J. 225

Ma, W. 235

Maity, D. 189

Majid, S. R. 195

Marzec, J. 91, 97

Mirsaneh, M. 25

Miyazaki, S. 209

Molenda, J. 91, 97

Moos, R. 127

Nakamura, Y. 19

Nakashima, S. 19

Nam, J. M. 145

Nam, T. H. $145,203,209$

Ohuchi, F. 177

Okuyama, M. 19

Ozarowski, A. 7

Pan, H. 1

Pan, H. L. 1

Park, W. 65

Popov, B. N. 155

Ramakrishna, S. 221

Ratinac, K. R. 55

Reaney, I. M. 25

Ree, T. V. 139

Ren, X. 167
Richter, T. 127

Ricinschi, D. 19

Riess, I. 105

Ringer, S. P. 55

Sato, T. 173

Schuh, C. 127

Shen, Z. 1

Shim, W. K. 225

Shon, J. K. 151

Shu, C. 121

Shuhaimi, N. E. A. 195

Si, Y. 167

Sikha, G. 155

So, B. G. 151

Soh, A. K. 59

Sun, C. 167

Suvaci, E. 127

Tatsumisago, M. 31

van Tol, J. 7

Wang, J. 221

Wang, X. 71

Wen, C. 215

Wouterson, E. 225

Wu, G. H. 115

Wu, P. 121

Wu, Y. P. 139

Xiao, Q. Z. 139

Xiong, J. 215

Xu, H. B. 115

$\mathrm{Xu}$, J. 167,247

Xue, D. 37, 167

Xue, J.-M. 189

Yan, C. $\quad 37,167$

Yang, S. H. 253

Yeo, C. Y. 225

Yimnirun, R. 133, 229

Yin, S. 173

Zalinska, B. 25

Zeng, H. C. 43

Zhang, H. P. 139

Zhang, H. W. 115

Zhang, P. 139 
Zhang, Q. 13

Zhang, X. 167

Zhang, Y. X. 43

Zhang, Z. 121
Zhao, X. 167

Zhao, X. B. 253

Zheng, G. P. 59

Zheng, R. 55
Zheng, R. Y. 221

Zhu, T. J. 253

Zhu, X. X. 115

Zhu, Y. C. 239 